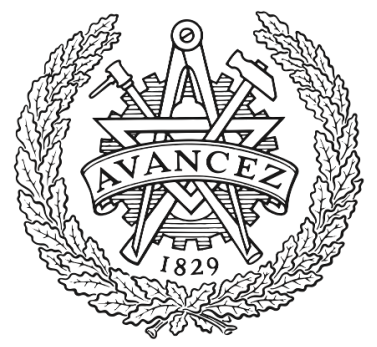

CHALMERS

UNIVERSITY OF TECHNOLOGY

\title{
Interplay between Cocaine, Drug Removal, and Methylphenidate Reversal on Phospholipid Alterations in Drosophila Brain Determined by Imaging
}

Downloaded from: https://research.chalmers.se, 2023-04-26 12:06 UTC

Citation for the original published paper (version of record):

Hoang Philipsen, T., Phan, N., Fletcher, J. et al (2020). Interplay between Cocaine, Drug Removal, and Methylphenidate Reversal on Phospholipid Alterations in Drosophila Brain Determined by Imaging Mass Spectrometry. ACS Chemical Neuroscience, 11(5): 806-813. http://dx.doi.org/10.1021/acschemneuro.0c00014

N.B. When citing this work, cite the original published paper. 


\title{
Interplay between Cocaine, Drug Removal, and Methylphenidate Reversal on Phospholipid Alterations in Drosophila Brain Determined by Imaging Mass Spectrometry
}

\author{
Mai Hoang Philipsen, Nhu T. N. Phan, John Stephen Fletcher, and Andrew G. Ewing*
}

Cite This: ACS Chem. Neurosci. 2020, 11, 806-813

Read Online

ACCESS | Lلll Metrics \& More | 回 Article Recommendations

SI Supporting Information

ABSTRACT: Cocaine dependence displays a broad impairment in cognitive performance including attention, learning, and memory. To obtain a better understanding of the action of cocaine in the nervous system, and the relation between phospholipids and memory, we have investigated whether phospholipids recover in the brain following cocaine removal using the fly model, Drosophila melanogaster. In addition, the effects of methylphenidate, a substitute medication for cocaine dependence, on fly brain lipids after cocaine abuse are also determined to see if it can rescue the lipid changes caused by cocaine. Time of flight secondary ion mass spectrometry with a $\left(\mathrm{CO}_{2}\right)_{6000}{ }^{+}$gas cluster ion beam was used to detect intact phospholipids. We show

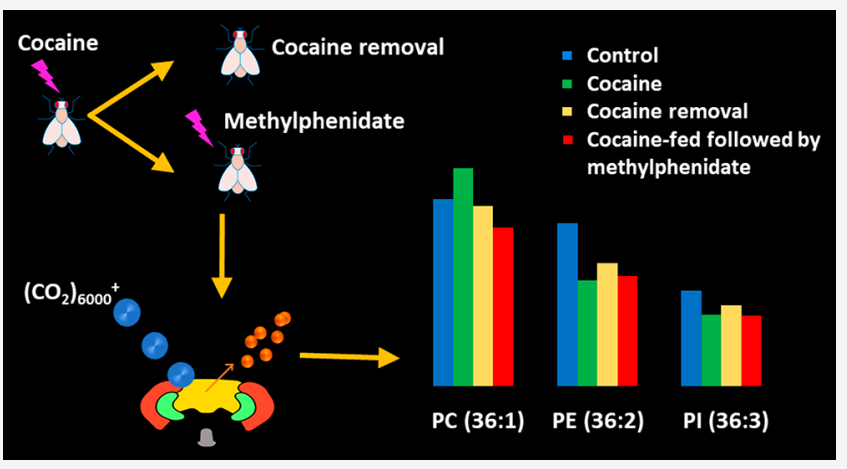
that cocaine has persistent effects, both increasing and decreasing the levels of specific phosphatidylethanolamines and phosphatidylinositols. These changes remain after cocaine withdrawal and are not rescued by methylphenidate. Cocaine is again shown to generally increase the levels of phosphatidylcholines in the fly brain; however, after drug withdrawal, the abundance of these lipids returns to the original level and methylphenidate treatment of the flies following cocaine exposure enhances the reversal of the lipid level reducing them below the original control. The study provides insight into the molecular effects of cocaine and methylphenidate on brain lipids. We suggest that phosphatidylcholines could be a potential target for the treatment of cocaine abuse as well as be a significant hallmark of cognition and memory loss with cocaine. KEYWORDS: Mass spectrometry imaging, lipids, cocaine removal, methylphenidate, Drosophila

\section{INTRODUCTION}

Cocaine addiction is a worldwide serious health problem. One of the primary mechanisms of cocaine action is its ability to block presynaptic dopamine transporters which results in an increase in dopamine levels in the synaptic cleft resulting in euphoria and reinforcement of the administration of cocaine. Once the dopamine transporters are blocked by cocaine, most of the dopamine in the synaptic cleft cannot be recycled via reuptake. ${ }^{1}$ Consequently, long-term repeated cocaine exposure inhibits dopamine transporters and consequently causes depletion of dopamine concentration and dysregulation of dopamine signaling in the brain. ${ }^{2}$ Neurochemical actions of cocaine also lead to an increase or a decrease in intracellular activities, especially the synthesis and degradation of phospholipids, in the reward-related brain regions which are associated with learning and memory. ${ }^{3}$ These changes in the brain might contribute to the deficit of brain functions observed including memory and cognition loss. Intriguingly, there is increasing literature showing that the symptoms of cognitive impairment are persistent long after cocaine withdrawal. ${ }^{4}$ However, there is still no effective medication available for cocaine abuse to date. One of the pharmacother- apeutic approaches for cocaine treatment is agonist replacement therapy in which methylphenidate $(\mathrm{MPH})$, for instance, can be used as a potential substitution for cocaine to reduce the cycle of compulsive use of cocaine. MPH also inhibits dopamine reuptake which induces drug addiction; however, its clearance in the brain is slower and therefore the abuse potential is lower compared to cocaine. MPH has been shown to exert opposite effects to cocaine on cognitive function; noticeably, it helps improve focus and memory for attention deficit hyperactivity disorder (ADHD) patients. Therefore, $\mathrm{MPH}$ might be a good candidate for treatment of cognition deficit caused by cocaine abuse.

Phospholipids are highly abundant in the brain and play important roles in brain function. Specifically, phospholipids regulate membrane trafficking, membrane fusion during exocytosis and endocytosis, regulate neurotransmitter recep-

Received: January 7, 2020

Accepted: February 11, 2020

Published: February 11, 2020 
tors for neuronal signaling and transmission, and supply energy for brain activity. ${ }^{5}$ Phospholipids are composed of different groups with specific types of headgroups and tails, which determines their physical and chemical properties as well as their shapes and morphologies. These properties in turn result in different effects of the lipids on the activities of other neuronal molecules, for example, alteration of presynaptic protein interactions, activity of ion channels, and membrane curvature and fluidity. ${ }^{6}$ Phosphatidylcholines (PCs) and cholesterol have been shown to diminish in the cellular membrane of PC12 cells following exposure to cisplatin, and this is suggested as a molecular mechanism underlying the change of neurotransmitter release by this drug. ${ }^{7}$ In addition, various neurological disorders and brain diseases such as Alzheimer's, ischemic stroke, $\mathrm{ADHD}$, and schizophrenia have been shown to result in alteration of brain lipids regarding their localization, abundance, and metabolism. ${ }^{8-12} \mathrm{MPH}$, as a common drug for $\mathrm{ADHD}$ patients, has been shown to change the lipid structure of the Drosophila melanogaster brain, especially by decreasing the abundance of cylindrical shaped PCs, and increasing the amount of conical shaped phosphatidylethanolamine (PEs) and phosphatidylinositols (PIs). ${ }^{13}$ Furthermore, from our previous study comparing the effects of cocaine and MPH on the lipid structure of the fly brain, ${ }^{14}$ it is apparent that these two drugs lead to opposite changes in differing phospholipid levels. In that work, we hypothesized this as a possible mechanism for the opposite effects of cocaine and MPH on learning and memory.

Mass spectrometry imaging (MSI) is a powerful tool for spatial interrogation of altered lipid biochemistry as a result of disease or exposure to exogenous compounds such as cocaine. $^{15,16}$ One of the MSI techniques, time-of-flight secondary ion mass spectrometry (ToF-SIMS), has been increasingly used in bioimaging to explore the lipid molecular architecture of cells and tissues. ${ }^{17}$ Given the high complexity and heterogeneity of the brain, it is essential to obtain detailed spatial organization in order to gain the insight of the relations between the molecular structure and function of the brain. ToF-SIMS offers the advantage that various molecules can be analyzed in parallel to obtain rich spatial molecular information inside the brain. The use of a high energy gas cluster ion beam (GCIB) with ToF-SIMS makes it particularly well suited for imaging intact phospholipids owing to the reduced fragmentation that occurs during sputtering by large primary ion clusters which therefore preserves the molecular ions. ${ }^{18,19}$

In this paper, we use ToF-SIMS imaging to test the hypothesis that the withdrawal of cocaine or addition of $\mathrm{MPH}$ after drug in Drosophila brain reverses the dramatic changes in the brain lipid composition caused by exposure to cocaine. We have investigated if this strategy can be used to rescue the adverse effects of cocaine on lipid composition and brain functions. Interestingly, we find that cocaine removal as well as $\mathrm{MPH}$ treatment after cocaine only partially reverse the phospholipid changes in the brain caused by cocaine. This treatment seems to amplify the effects of cocaine removal on PCs and partially on triacylglycerols (TAGs), but neither cocaine removal nor $\mathrm{MPH}$ alters the levels of PE and PI species determined to change after cocaine. These seem to be more permanent changes that cannot be easily rescued.

\section{RESULTS AND DISCUSSION}

ToF-SIMS is a powerful approach for studying lipidomic profiles and distributions in biological tissues. Due to the use of the relatively new GCIB technology, ion sources such as $\mathrm{Ar}_{4000}{ }^{+}$and $\left(\mathrm{CO}_{2}\right)_{6000}{ }^{+}$provide greatly enhanced sputter yields of high mass molecular ions. Thus, a variety of intact phospholipids can be detected in fly brain sections. In order to evaluate the long-term effect of cocaine withdrawal or follow up $\mathrm{MPH}$ treatment on the phospholipid structure of the brain, we used ToF-SIMS with a $\left(\mathrm{CO}_{2}\right)_{6000}{ }^{+} \mathrm{GCIB}$ to image phospholipid localization in fly brain sections after the different drug treatments. One group of flies was treated with cocaine for 3 days and then fed with normal yeast-based food for 3 more days. Another set of cocaine-treated flies was subsequently treated by oral administration of $\mathrm{MPH}$ for 3 days. Comparison of the ion images of individual phospholipid species indicates a clear change in their distribution after cocaine removal or $\mathrm{MPH}$ treatment. In the positive ion mode, in the control flies, PCs, for instance PC (36:1), are mostly located in the central brain and some parts of optical lobe, especially the lobula complex (Figure 1A). Interestingly, the

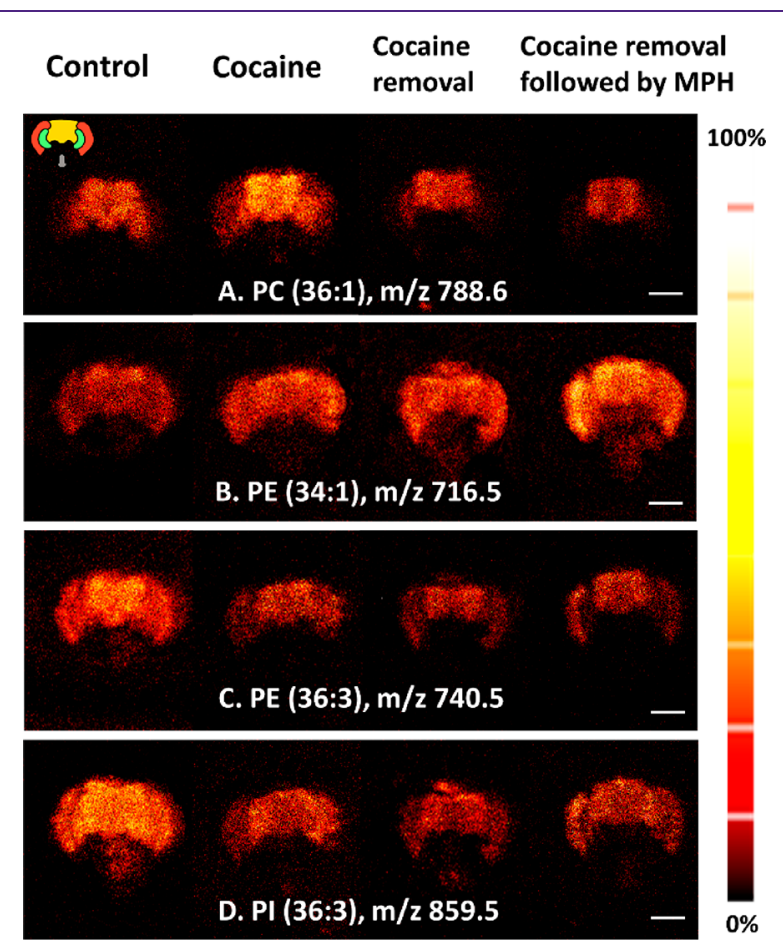

Figure 1. ToF-SIMS ion images show accumulation of phospholipids in fly head analyzed by ToF-SIMS with a $40 \mathrm{keV}\left(\mathrm{CO}_{2}\right)_{6000}{ }^{+}$GCIB. (A) PC (36:1), $[\mathrm{M}+\mathrm{H}]^{+}$, in positive ion mode, (B, C) PE (34:1) and PE (36:3), $[\mathrm{M}-\mathrm{H}]^{-}$, in negative ion mode, (D) PI (36:3), [M $\mathrm{H}]^{-}$, in negative ion mode. Primary ion dose density $1.7 \times 10^{13}$ ions/ $\mathrm{cm}^{2}$. Image area is $1000 \times 1000 \mu \mathrm{m}^{2}$ and $256 \times 256$ pixels. Scale bar is $200 \mu \mathrm{m}$. Signal intensity is displayed on a color thermal scale on the right panel. Symbolic figure on the top left shows the orientation of the fly head section: two red parts are medullas of optical lobes, two green parts are optical lobula, central brain is the yellow part in the middle, and gray part is proboscis.

PC species spread out to the medulla region of the optical lobe in the cocaine-treated brain, whereas after cocaine removal their distributions return to those observed in the control group. In addition, the regional intensity of PC species increases in abundance mostly in the central brain after cocaine and then decreases to control levels after cocaine removal, but it shows even lower abundance and is more focused in the 
central brain region of cocaine-fed flies when followed by $\mathrm{MPH}$ administration during cocaine removal.

In the negative ion mode, the data show that PE and PI species are distributed more evenly across the whole control brain, while showing slightly higher intensity in the central brain and optical lobe regions. After cocaine administration, there are different trends in the distributions. For example, an enhancement of PE (34:1) intensity is observed in the cocainetreated flies compared to the control flies. This is more or less uniform across the brain, but it appears a little stronger in the optical lobes. This increase remains at the same level after the flies are removed from cocaine exposure for 3 days, and the abundance is even more pronounced after being treated by $\mathrm{MPH}$ following the cocaine (Figure 1B). In contrast to PE (34:1), unsaturated PEs and PIs with 36 carbons in the fatty acid chains are decreased in the central brain and the optical lobe in the cocaine-fed fly brains compared to control flies. These also remain unchanged after cocaine removal, or after $\mathrm{MPH}$ treatment (Figure 1C, D), although PI, such as PI (36:3), appears to recover slightly in the brains treated with $\mathrm{MPH}$ during cocaine recovery (semiquantitative data are given below). Overall, the data show that cocaine removal and MPH treatment after cocaine exposure reverse the effects of cocaine on the PC distributions in the fly brain, whereas there is no significant reversal in the changes in the perturbed PE and PI lipids caused by cocaine when it is removed or the flies are exposed to MPH during the recovery time.

Previous work showed that the effects of cocaine on the fly brain were most prevalent in the alteration of phospholipid compositions mainly in the central brain area, but no significant changes were observed in the optical regions and proboscis. ${ }^{14}$ In the current study, all the peaks in the spectra have been collected from the central regions of the fly brain, and to elucidate the chemical changes principal components analysis (PCA) was performed on ToF-SIMS spectra. These were normalized to the number of selected pixels and total ion counts. The score plot of PCA from the extracted data clearly distinguishes three different groups along the second and third principal components.

Figure 2A shows a clear separation in principal component 3 for the control and cocaine removal groups from the drugtreated groups. In Figure 2B, the loadings plot of principal component 3 is included showing which peaks contribute most to the separation observed in the score plot of principal component 3 . The separation is mainly caused by peaks in the intact lipid region (e.g., TAG and PC species). The assignment of peaks, which show significant alterations in principal component 3, can be found in Table S1. From Figure 2B, PC species, for example PC (36:1) at $m / z 788.6$, PC $(34: 1)+$ $\mathrm{K}$ at $m / z 798.5$, and $\mathrm{PC}(36: 3)+\mathrm{K}$ at $m / z 822.5$, are more abundant in the purely cocaine-fed group and the group fed cocaine followed by MPH treatment. Likewise, other fragments of PC salt adducts such as PC $(34: 2)+\mathrm{Na}$-TMA at $\mathrm{m} / z$ 721.5 and PC $(36: 2)+$ Na-TMA at $m / z 749.5$, where TMA $(59 \mathrm{Da})$ is the trimethylamine group at the end of the PC headgroup, display more intense intensity in cocaine-fed flies and cocaine-fed flies followed by MPH treatment. In contrast, elevations of the levels of TAG salt adducts, such as TAG $(48: 1)+\mathrm{Na}$ at $m / z 827.7$, TAG $(48: 1)+\mathrm{K}$ at $m / z 843.7$, and TAG $(50: 1)+\mathrm{K}$ at $m / z 871.7$, are observed in control brains and those after cocaine removal. The diacylglycerol (DAG) peaks in the mass range $m / z 400-650$ are probably the fragments from TAGs and other lipids; hence, we did not
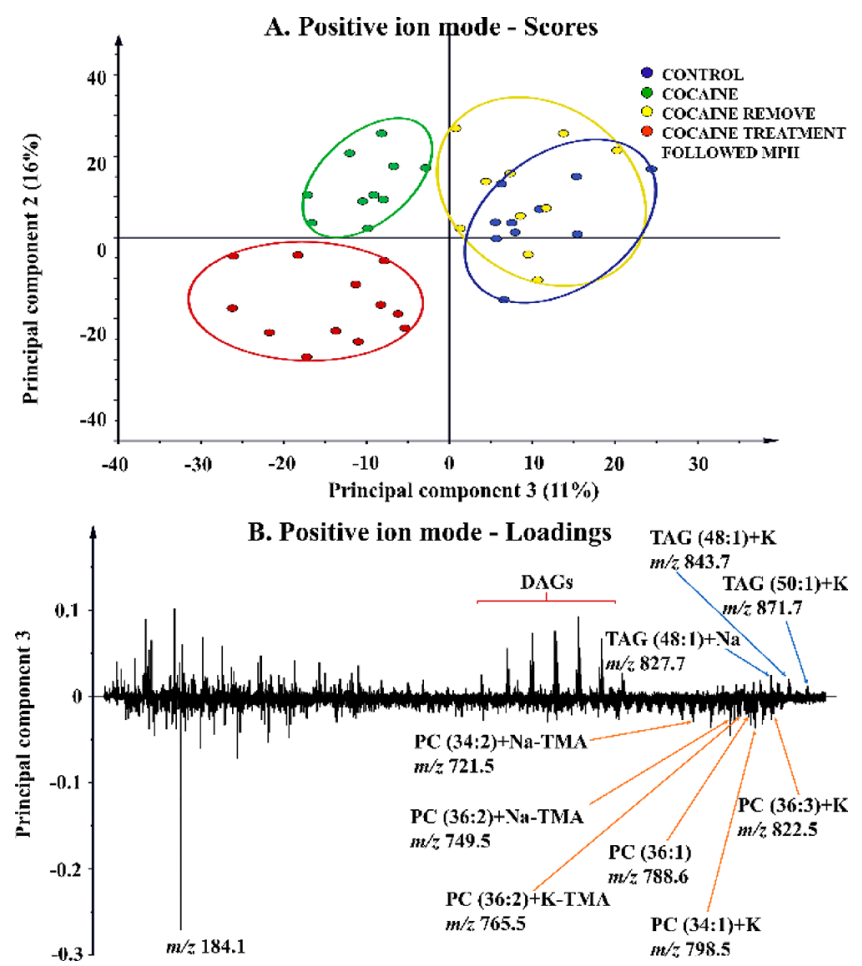

Figure 2. PCA for ToF-SIMS data in positive mode. (A) Score plot of the second principal component versus the third principal component; (B) corresponding loadings for the third principal component. The ellipse on the score plot calculated from Hotelling's T-square statistic is related to the $95 \%$ confidence interval.

attempt to identify these species. In addition, use of principal component 2 separates the cocaine-fed group from the cocaine-fed followed by MPH group (Figure S1). Specifically the PCA results suggest that the control, cocaine-fed, and cocaine removal groups have higher abundances of PCs and their salt adducts, such as PC (34:1) at $m / z$ 760.6, PC (36:2) $+\mathrm{K}$ at $m / z$ 824.6, and PC (36:2) + K-TMA at $m / z$ 765.5, compared to the cocaine-fed group followed by $\mathrm{MPH}$ treatment. The signal level for the PC headgroup at $\mathrm{m} / \mathrm{z}$ 184.1, which is correlated with PC species, is also decreased in cocaine-treated flies followed by MPH during the cocaine removal time. In contrast, the flies fed cocaine followed by $\mathrm{MPH}$ treatment during the cocaine removal time have spectra that are more dominant in the content of TAGs, for example, TAG $(48: 1)+\mathrm{K}$ at $m / z$ 843.7, TAG $(50: 2)+\mathrm{Na}$ at $m / z$ 853.7, and TAG $(52: 2)+\mathrm{Na}$ at $m / z 881.8$.

While the PCA analysis can indicate which species may be related to the separation of the peaks the nature of the scores and loadings output can make precise interpretation difficult. Hence it is critical that one returns to the raw data for further validation. Therefore, to obtain detailed information about the alterations in phospholipid compositions in fly brains after drug treatments and removal, the lipid species identified by PCA were selected and their relative concentrations were compared among different groups. Figure 3 shows that there are significant changes in PC levels after drug treatment compared to control. PCs detected as $[\mathrm{M}+\mathrm{H}]^{+}$and their salt adducts are increased in the fly brains after cocaine administration. The elevation of PC abundance is mainly observed in PC species with 32, 34, and 36 carbon fatty acid chains and different saturation levels, such as PC (32:1) at $m / z$ 732.6, PC (32:0) at $m / z$ 734.6, PC (34:2) at $m / z 758.6$, PC 


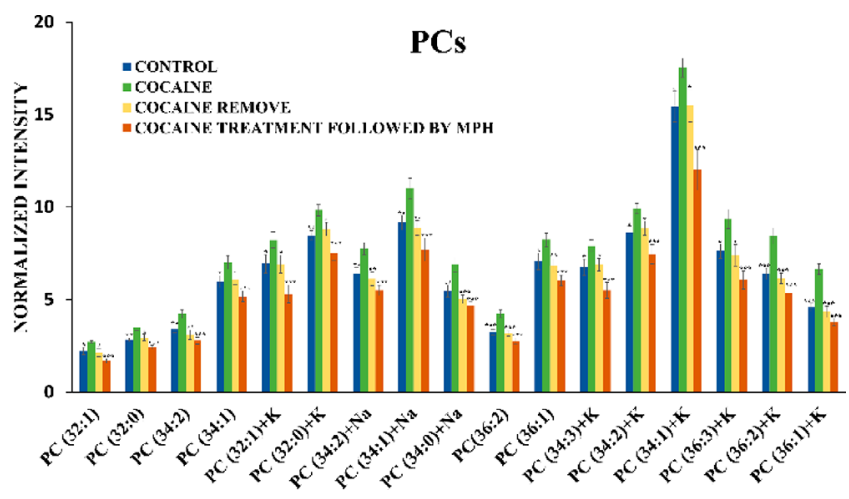

Figure 3. Changes in phosphatidylcholine levels in the central fly brain after cocaine and cocaine removal, and in flies with cocaine treatment followed by MPH oral administration in positive ion mode analyzed using a $40 \mathrm{keV}\left(\mathrm{CO}_{2}\right)_{6000}{ }^{+}$GCIB. Peak intensity is normalized to the number of pixels selected and total signal intensity. Error bars are standard error of the mean of 33 controls (blue bars), 28 cocaine-treated flies (green bars), 27 flies with cocaine withdrawal (yellow bars), and 27 flies with MPH feeding after cocaine treatment (red bars). A $t$ test was used to compare the statistical difference between the cocaine group with the other groups $\left({ }^{*} p<0.5\right.$, ** $p<$ $0.01, * * * p<0.001)$. No statistical differences were found between control and cocaine-removed groups $(p>0.05)$. Ions were detected as $[\mathrm{M}+\mathrm{H}]^{+}$unless specified as $\mathrm{Na} / \mathrm{K}$ adduct species.

(34:1) at $m / z 760.6, \mathrm{PC}(36: 2)$ at $m / z 786.6$, and PC (36:1) at $\mathrm{m} / z$ 788.6. After cocaine removal, the levels of these PC species in the central brain return to the same level as in the control brains. MPH treatment during the removal time after cocaine exposure, however, induces further depletion in the PC levels compared to the control brains, which is consistent with observations in our previous reports ${ }^{13,14}$ and the images in Figure 1.

The relative amounts of TAGs in different fly brain groups are shown in Figure S2. Cocaine induces a dramatic decrease in TAG levels, and these recover partially after cocaine removal. MPH treatment after cocaine exposure and removal leads to recovery of the TAG levels to an even greater extent, but still lower than those in the control. Based on these data, we conclude that cocaine removal leads to recovery of the effects of cocaine on the abundance of PCs, and MPH further reverses the effects of cocaine on these phospholipids to an extent past control values. In addition, both treatment strategies can also partially rescue the change in TAG concentrations in fly brains following cocaine exposure.

We observe that PC levels are increased in the central fly brain following cocaine exposure. An elevation of PC abundance was also observed in rat brains treated with other stimulants which also induces the impairment of cognitive performance. $^{20}$ There are now several pieces of evidence suggesting that brain phospholipids, especially PCs, are involved in brain function and mental performance. ${ }^{21,22}$ PCs are closely related to the production of choline which is a precursor for acetylcholine, a neurotransmitter that plays many biological roles in developmental and cognitive function. ${ }^{23} \mathrm{PC}$ levels decrease again to normal levels in fly brains after cocaine removal or after $\mathrm{MPH}$ treatment. In addition to $\mathrm{ADHD}$ patients, cognitive enhancement following MPH has also been reported for patients with traumatic brain injury as well as depression after stroke. ${ }^{24-26}$ Moreover, Goldstein's group showed that the use of a low dose of MPH improved cognitive tasks in cocaine abusers. ${ }^{27}$ It is enticing to speculate that the changes we observe in lipids following cocaine and those rescued by $\mathrm{MPH}$ are in some way involved in these cognitive changes. It should be noted that incubation of cells with lipids changes the rate of exocytosis. ${ }^{28,29}$

PCA was carried out on ToF-SIMS spectra in the negative ion mode to elucidate the difference for the recovery of PEs and PIs between the control and drug-treated groups. The PCA score plot of principal component 1 versus principal component 2 , which together capture $58 \%$ of the total variance in the data set, is shown in Figure 4. The control group is
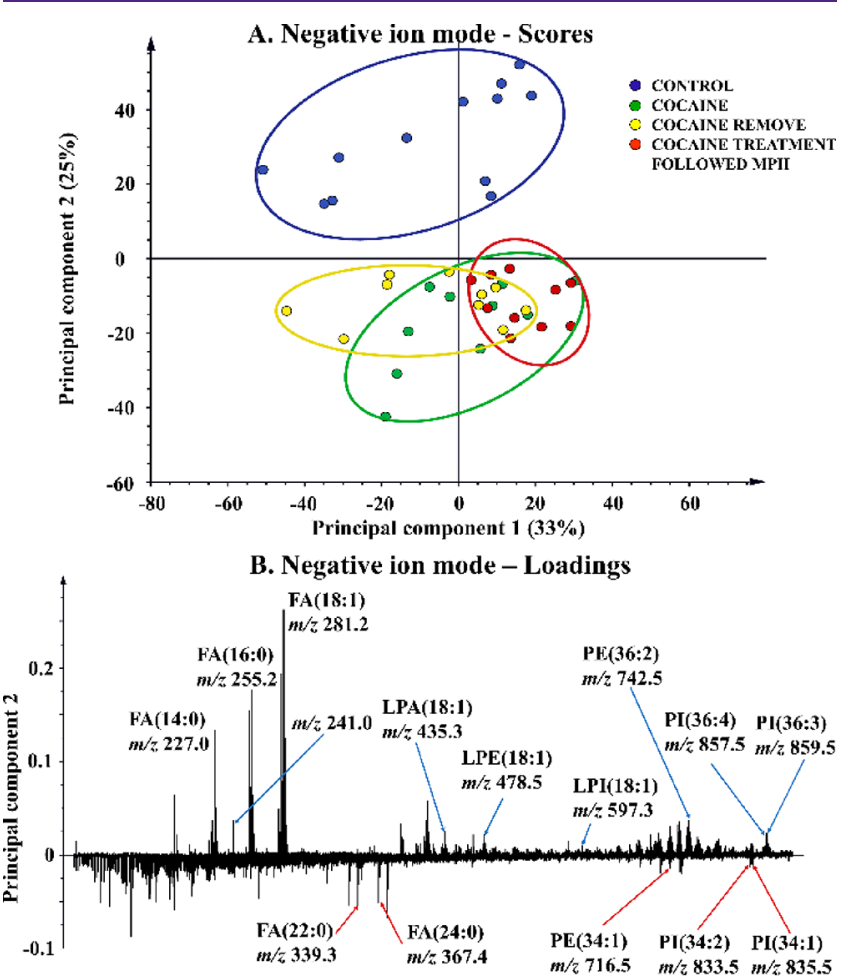

Figure 4. PCA for ToF-SIMS data in negative mode. (A) Score plot of the first principal component versus the second principal component; (B) corresponding loading plot for the second principal component. The ellipse on the score plot calculated from Hotelling's $\mathrm{T}$-square statistic is related to the $95 \%$ confidence interval.

clearly separated from the others across principal component 2, whereas all the drug treated groups, including the cocaine exposure, cocaine removal, and cocaine exposure followed by $\mathrm{MPH}$ groups, overlap in the scores plot (Figure 4A).

The peaks contributing to the difference between the control and the other groups in the PCA can be identified by the loading plot of principal component 2 (Figure 4B). The significant lipids that are decreased in the drug-treated brains versus control are PE/PI species such as $\mathrm{PE} \mathrm{(36:2)} \mathrm{at} \mathrm{m} / z$ 742.5, PI (36:4) at $m / z 857.5$, and PI (36:3) at $m / z 859.5$, lysolipids such as lysophosphatidic acid LPA (18:1) at $\mathrm{m} / z$ 435.3, lysophosphatidylethanolamine LPE (18:1) at $\mathrm{m} / z$ 478.3, and lysophosphatidylinositol LPI $(18: 1)$ at $m / z$ 597.3, and fatty acids, for example, FA $(18: 1)$ at $m / z 281.2$ and FA (16:0) at $m / z$ 255.2. In contrast, several PEs and PIs with 34 carbon fatty acid chains, such as PE $(34: 1)$ at $m / z 716.5$, PI $(34: 2)$ at $m / z 833.5$, and PI $(34: 1)$ at $m / z 835.5$, as well as fatty acids FA (22:0) at $m / z 339.3$ and FA (24:0) at $m / z 367.4$ are increased in the drug-treated groups. 
After cocaine administration, there is an accumulation of PEs and PIs with 34 carbons in the fatty acid chain and a reduction of PEs and PIs with 36 carbons as well as several LPA, LPE, and LPI species (Figure S3). PCA, however, shows no separation between the cocaine administration, cocaine removal groups, and cocaine-fed flies followed by $\mathrm{MPH}$ treatment. The effect of cocaine on PE and PI phospholipids, and several lipid precursors in the fly brain is still persistent even after the flies are removed from the drug as well as treated with MPH. This is also supported by the comparison of the relative abundances of various significant lipid species in the fly brains undergoing different drug treatments (Figure 5). There
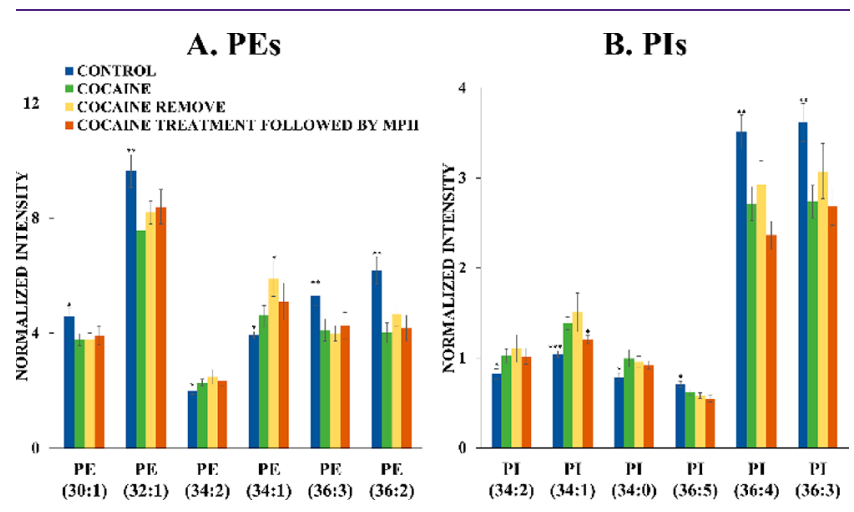

Figure 5. Relative amounts of phospholipids in the central fly brain after cocaine, cocaine removal, and flies with cocaine treatment followed MPH oral administration in negative ion mode analyzed using a $40 \mathrm{keV}\left(\mathrm{CO}_{2}\right)_{6000}{ }^{+}$GCIB. (A) PE levels; (B) PI levels. Peak intensity is normalized to number of pixels selected and total signal intensity. Error bars are standard error of the mean 32 controls (blue bars), 24 cocaine-treated flies (green bars), 26 flies with cocaine withdrawal (yellow bars), and 24 flies fed with cocaine followed by MPH (red bars). A $t$ test was used to compare the difference between the cocaine group and the other groups $\left(* p<0.5,{ }^{*} p<0.01, * * * p<\right.$ $0.001)$. There are no statistical differences between cocaine groups and the cocaine-removed groups and cocaine-treated followed by MPH group $(p>0.05)$. All ions were detected as $[\mathrm{M}-\mathrm{H}]^{-}$.

is a significant increase in PEs and PIs with 34 carbon fatty acid chains, including PE (34:2), PE (34:1), PI (34:2), PI (34:1), and PI (34:0), after cocaine administration and persisting after cocaine removal and $\mathrm{MPH}$ treatment. In contrast, cocaine exposure decreases the amount of several other lipids, for instance, $\mathrm{PE}(30: 1)$ at $m / z 660.5, \mathrm{PE}(32: 1)$ at $m / z 688.5, \mathrm{PE}$ (36:3) at $m / z 740.5, \mathrm{PE}(36: 2)$ at $m / z 742.5$, PI $(36: 5)$ at $m / z$ 855.5, PI (36:4) at $m / z 857.5$, and PI (36:3) at $m / z 859.5$, and, again, the withdrawal of cocaine does not recover the effect of cocaine on these lipids. Similarly, these PE and PI levels stay the same when flies are subsequently treated with $\mathrm{MPH}$ for 3 days. Overall, cocaine significantly alters the levels of PEs and PIs and these changes are not reversible by cocaine removal and subsequent treatment with $\mathrm{MPH}$, at least for 3 days.

Oral administration cocaine results in significant changes in the phospholipid composition of the fly brain, consistent with previous results. ${ }^{14}$ PCs and the 34-carbon chain PEs and PIs are increased, and total PEs and PIs are decreased after cocaine exposure. Cocaine administration has been shown to decrease the activity of phospholipase $\mathrm{A}_{2}\left(\mathrm{PLA}_{2}\right)$ in the rat brain striatum due to the increase in dopamine levels. ${ }^{30}$ A schematic of the metabolic pathways of phospholipids is provided in Figure S4. PLA 2 is the enzyme responsible for the degradation of phospholipids at the sn-2 position to release 1-acyl lysophospholipids and free fatty acids. ${ }^{31}$ Reduced $\mathrm{PLA}_{2}$ activity caused by cocaine might be associated with the elevation of phospholipids, mainly PCs and some PEs and PIs, observed. In addition, the inhibition of $\mathrm{PLA}_{2}$ induced by psychostimulants such as cocaine might result in the depletion of lysophospholipids in the fly brain as it is observed in the reduction of LPA (18:1), LPE (18:1), and LPI (18:1) in the cocaine-treated flies (Figure 4B). However, the concentrations of PCs in the fly brain return to normal values after the flies were removed from cocaine. Thus, if the $\mathrm{PLA}_{2}$ enzyme is responsible for these increased lipid levels, then it is reversible and its activity is recovered after cocaine removal. ${ }^{30}$

Phospholipase C (PLC) is a membrane-associated enzyme that promotes the conversion of PIs to DAGs. Cocaine has been found to stimulate PLC activity via the activation of $\mathrm{G}_{\mathrm{q} / 11 \text {-coupled receptors. }}{ }^{32}$ PLC activity has also been found to be increased in the frontal cortex of rats after withdrawal from repeated administration of cocaine. ${ }^{33,34}$ As the activity of PLC increases, PIs are degraded into diacylglycerol and secondary messenger molecules such as inositol 1,4,5-trisphosphate. Hence, we propose that the cocaine has long-term effects on the activity of PLC, which in turn affects the phospholipids in the long term even after withdrawal. Importantly, these persistent alterations on PEs and PIs could be associated with the cognitive deficits observed long after cocaine withdrawal. Several reports have shown that dysregulation in cognition and emotion is persistent in subjects even after cocaine withdrawal, and also after other stimulants. ${ }^{4,35,36}$ Since our data show that the depletion of PEs and PIs remain after cocaine removal, these changes might be associated with the cognitive impairment caused by cocaine.

An important goal for pharmacotherapeutic medicine of drug abuse is a therapeutic treatment for cocaine addiction. Several studies have suggested that MPH can be used as an agonistic substitute for treatment of cocaine dependence owing to their similarity in structure and basic mechanism in blocking dopamine uptake. However, although cocaine and methylphenidate have similar effects in blocking dopamine reuptake, they have opposite effects on their changing the phospholipid composition in fly brain. ${ }^{14}$ Moreover, it has been reported by Calipari et al. that cocaine and methylphenidate have opposite effects on the dopaminergic system. ${ }^{37}$ Cocaine decreased dopamine transport and dopamine uptake rate and increased the dopamine release, whereas MPH administration increased dopamine transporter density, dopamine uptake rate, and dopamine release. Our data (Figure 3) reveal that PC abundance in the fly brain has a tendency to increase after cocaine administration; however, there is a downward trend or reversal when cocaine is removed, and this downward trend is exaggerated when the cocaine-fed flies are subsequently treated with $\mathrm{MPH}$. In contrast, the changes in PEs and PIs in the fly brain after cocaine exposure are not reversed with cocaine removal or MPH treatment. Zhu et al. found that cocaine and $\mathrm{MPH}$ cause opposite effects on the release fraction during exocytosis from PC12 cells. ${ }^{38}$ They suggested that cocaine stimulated protein kinase $C$, which then synthesized filamentous actin and consequently increased the rate of the closing of the fusion pore during open and closed exocytosis. Unlike cocaine, MPH, which shows no effects on actin immunoactivity, ${ }^{39}$ shows normal exocytosis similar to control cells. Thus, a possible mechanistic aspect to the effects of cocaine and $\mathrm{MPH}$ might be that they induce various effects on 
membrane phospholipids, which then link to the stability of the fusion pores for exocytosis.

In summary, PCs, PEs, and PIs might be considered to be therapeutic targets for treatment of cognitive deficit caused by cocaine. Although cocaine removal and $\mathrm{MPH}$ treatment after cocaine removal do not completely reverse the dramatic alterations of all phospholipids in the brain caused by cocaine, they impose a significantly positive effect on the adverse action of cocaine on PCs and partially on TAGs. The approaches presented here might be further examined to develop rescue strategies for cognitive impairment by cocaine addiction.

\section{METHODS}

Chemicals. All chemicals were purchased from Sigma-Aldrich, Sweden.

Drosophila Preparation. Wide type Drosophila strain, Canton-S, was maintained in standard cornmeal food at room temperature (23$26{ }^{\circ} \mathrm{C}$ ) with a $12 \mathrm{~h} \mathrm{light/dark} \mathrm{cycle.} \mathrm{Three} \mathrm{to} \mathrm{four} \mathrm{day} \mathrm{old} \mathrm{male} \mathrm{flies}$ were selected and fed with yeast paste food supplemented with cocaine to get the final concentration to $15 \mathrm{mM}$ for 3 days. Cocainefed flies were divided into three groups: the first group had continued cocaine feeding for another 3 days; the second group, the so-called cocaine removal group, was subsequently fed with normal yeast paste food without cocaine for 3 days; and the third group was orally administered with food containing $10 \mathrm{mM} \mathrm{MPH}$ for 3 days after cocaine administration. The feeding dose of $10 \mathrm{mM} \mathrm{MPH}$ to flies in vivo corresponds to a dose of $4.8 \mathrm{mg} / \mathrm{kg}$ which is in the range used for a recent pharmacokinetics and bioavailability study in rats $(0.75-10$ $\mathrm{mg} / \mathrm{kg}) .^{40,41}$ After drug treatment, flies from different groups were placed sequentially on the same fly collars (4 M Instrument \& Tool LLC) and embedded in $10 \%$ gelatin. ${ }^{13}$ The gelatin molds were frozen at $-20{ }^{\circ} \mathrm{C}$ and then in liquid nitrogen where the fly-head-embedded gelatin was detached from the collar. The fly-head-embedded gelatin was subsequently cut at $12 \mu \mathrm{m}$ thickness using a cryo-microtome at $-20{ }^{\circ} \mathrm{C}$ (Leica CM1520). Sections were thaw-mounted on indium tin oxide coated glass and freeze-dried overnight in the ToF-SIMS instrument for further analysis.

ToF-SIMS Analysis: J105. ToF-SIMS analysis was performed using the J105-3D Chemical Imager (Ionoptika Ltd., UK). The principle operation of this instrument has been described in detail elsewhere. ${ }^{42}$ In our experiments, a GCIB of $40 \mathrm{keV}\left(\mathrm{CO}_{2}\right)_{6000}{ }^{+}$was used as a primary ion beam to sputter the sample surface in positive and negative ion modes. High energy clusters help enhance the primary and secondary ion yields for intact lipid signals in biological samples and allow detection of high mass species both on the sample surface and in the subsurface. ${ }^{19}$ The spectra were acquired over a mass range of $m / z 100-1000$. The image area was $1000 \times 1000 \mu \mathrm{m}^{2}$ with $128 \times 128$ pixels for statistical analysis, or with $256 \times 256$ pixels for imaging with higher spatial resolution, and the beam size was $6 \mu \mathrm{m}$ with a primary ion current of $17 \mathrm{pA}$, which resulted in the total ion dose density of $6.1 \times 10^{12}$ and $1.7 \times 10^{13}$ ions $/ \mathrm{cm}^{2}$, respectively. The samples were analyzed in the freeze-dried condition at room temperature.

Data Analysis. SIMCA (Umetrix, Sweden) was used to perform principal components analysis (PCA) on ToF-SIMS spectra which were extracted by selecting the region of interest, the central area of the fly brain, from ToF-SIMS images. All spectra were normalized to total number of pixels and total ion intensity of the region of interest to compensate for the variations from ToF-SIMS measurements. PCA was then applied to this data set after Pareto scaling. Pareto scaling used square root of standard deviation as a scaling factor to reduce the dynamic range in the data and emphasize weak peaks that might be more relevant to biological information.

A $t$ test was performed on the nonscaled peak intensities of the ions identified in the PCA loadings to compare the data from the cocaine group with the other groups.

\section{ASSOCIATED CONTENT}

Supporting Information

The Supporting Information is available free of charge at https://pubs.acs.org/doi/10.1021/acschemneuro.0c00014.

Table of lipid peak assignment, PCA analysis for ToFSIMS data in positive mode, relative abundance of TAG and lysophospholipids, and scheme of phospholipase activity (PDF)

\section{AUTHOR INFORMATION}

\section{Corresponding Author}

Andrew G. Ewing - Department of Chemistry and Chemical Engineering, Chalmers University of Technology, Gothenburg 412 96, Sweden; Department of Chemistry and Molecular Biology, University of Gothenburg, Gothenburg 412 96, Sweden; The Gothenburg Imaging Mass Spectrometry (Go:IMS) Platform, Gothenburg, Sweden; — orcid.org/0000-0002-20840133; Email: andrewe@chem.gu.se

\section{Authors}

Mai Hoang Philipsen - The Gothenburg Imaging Mass Spectrometry (Go:IMS) Platform, Gothenburg, Sweden

Nhu T. N. Phan - Department of Chemistry and Molecular Biology, University of Gothenburg, Gothenburg 412 96, Sweden; The Gothenburg Imaging Mass Spectrometry (Go:IMS) Platform, Gothenburg, Sweden

John Stephen Fletcher - Department of Chemistry and Molecular Biology, University of Gothenburg, Gothenburg 412 96, Sweden; The Gothenburg Imaging Mass Spectrometry (Go:IMS) Platform, Gothenburg, Sweden; ○ orcid.org/00000002-9418-8571

Complete contact information is available at: https://pubs.acs.org/10.1021/acschemneuro.0c00014

\section{Author Contributions}

All authors have given approval to the final version of the manuscript.

\section{Funding}

This work was supported by the Knut and Alice Wallenberg Foundation, the European Research Council (ERC), and the Swedish Research Council (VR).

\section{Notes}

The authors declare no competing financial interest.

\section{ACKNOWLEDGMENTS}

All experiments were performed at the go:IMS at Chalmers University of Technology/Gothenburg University (www.goims.gu.se).

\section{ABBREVIATIONS}

MSI, mass spectrometry imaging; ToF-SIMS, time-of-flight secondary ion mass spectrometry; GCIB, gas cluster ion beam; $\mathrm{MPH}$, methylphenidate; ADHD, attention deficit hyperactive disorder; PE, phosphatidylethanolamine; PC, phosphatidylcholine; PI, phosphatidylinositol; PCA, principal component analysis; TAG, triacylglycerol; DAG, diacylglycerol; LPE, lysophosphatidylethanolmine; LPA, lysophosphatidic acid; LPI, lysophosphatidylinositol; PLA 2 , phospholipase $\mathrm{A}_{2}$; PLC, phospholipase C 


\section{REFERENCES}

(1) Meiser, J., Weindl, D., and Hiller, K. (2013) Complexity of dopamine metabolism. Cell Commun. Signaling 11 (1), 34.

(2) McIntosh, S., Howell, L., and Hemby, S. E. (2013) Dopaminergic dysregulation in prefrontal cortex of rhesus monkeys following cocaine self-administration. Front Psychiatry 4, 88.

(3) Schneider, M., Levant, B., Reichel, M., Gulbins, E., Kornhuber, J., and Muller, C. P. (2017) Lipids in psychiatric disorders and preventive medicine. Neurosci. Biobehav. Rev. 76 (Pt B), 336-362.

(4) Kelley, B. J., Yeager, K. R., Pepper, T. H., and Beversdorf, D. Q. (2005) Cognitive impairment in acute cocaine withdrawal. Cogn Behav Neurol 18 (2), 108-112.

(5) Ammar, M. R., Kassas, N., Chasserot-Golaz, S., Bader, M. F., and Vitale, N. (2013) Lipids in Regulated Exocytosis: What are They Doing? Front. Endocrinol. (Lausanne, Switz.) 4, 125.

(6) Lauwers, E., Goodchild, R., and Verstreken, P. (2016) Membrane Lipids in Presynaptic Function and Disease. Neuron 90 (1), 11-25.

(7) Mohammadi, A. S., Li, X., and Ewing, A. G. (2018) Mass Spectrometry Imaging Suggests That Cisplatin Affects Exocytotic Release by Alteration of Cell Membrane Lipids. Anal. Chem. 90 (14), $8509-8516$.

(8) Burgess, J. R., Stevens, L., Zhang, W., and Peck, L. (2000) Longchain polyunsaturated fatty acids in children with attention-deficit hyperactivity disorder. Am. J. Clin. Nutr. 71 (1 Suppl), 327S-330S.

(9) Kidd, P. M. (2007) Omega-3 DHA and EPA for cognition, behavior, and mood: clinical findings and structural-functional synergies with cell membrane phospholipids. Altern. Med. Rev. 12 (3), 207-227.

(10) Adibhatla, R. M., and Hatcher, J. F. (2008) Altered lipid metabolism in brain injury and disorders. Subcell Biochem 49, 241268.

(11) Schwarz, E., Prabakaran, S., Whitfield, P., Major, H., Leweke, F. M., Koethe, D., McKenna, P., and Bahn, S. (2008) High throughput lipidomic profiling of schizophrenia and bipolar disorder brain tissue reveals alterations of free fatty acids, phosphatidylcholines, and ceramides. J. Proteome Res. 7 (10), 4266-4277.

(12) Lim, W. L., Martins, I. J., and Martins, R. N. (2014) The involvement of lipids in Alzheimer's disease. J. Genet. Genomics 41 (5), 261-274.

(13) Phan, N. T. N., Fletcher, J. S., and Ewing, A. G. (2015) Lipid structural effects of oral administration of methylphenidate in Drosophila brain by secondary ion mass spectrometry imaging. Anal. Chem. 87 (8), 4063-4071.

(14) Philipsen, M. H., Phan, N. T. N., Fletcher, J. S., Malmberg, P., and Ewing, A. G. (2018) Mass Spectrometry Imaging Shows Cocaine and Methylphenidate Have Opposite Effects on Major Lipids in Drosophila Brain. ACS Chem. Neurosci. 9 (6), 1462-1468.

(15) Kaya, I., Zetterberg, H., Blennow, K., and Hanrieder, J. (2018) Shedding Light on the Molecular Pathology of Amyloid Plaques in Transgenic Alzheimer's Disease Mice Using Multimodal MALDI Imaging Mass Spectrometry. ACS Chem. Neurosci. 9 (7), 1802-1817.

(16) Pati, S., Angel, P., Drake, R. R., Wagner, J. J., and Cummings, B. S. (2019) Lipidomic changes in the rat hippocampus following cocaine conditioning, extinction, and reinstatement of drug-seeking. Brain Behav 9 (12), No. e01451.

(17) Tian, H., Fletcher, J. S., Thuret, R., Henderson, A., Papalopulu, N., Vickerman, J. C., and Lockyer, N. P. (2014) Spatiotemporal lipid profiling during early embryo development of Xenopus laevis using dynamic ToF-SIMS imaging. J. Lipid Res. 55 (9), 1970-1980.

(18) Angerer, T. B., Blenkinsopp, P., and Fletcher, J. S. (2015) High energy gas cluster ions for organic and biological analysis by time-offlight secondary ion mass spectrometry. Int. J. Mass Spectrom. 377, 591-598.

(19) Dimovska Nilsson, K., Palm, M., Hood, J., Sheriff, J., Farewell, A., and Fletcher, J. S. (2019) Chemical Changes On, and Through, The Bacterial Envelope in Escherichia coli Mutants Exhibiting Impaired Plasmid Transfer Identified Using Time-of-Flight Secondary Ion Mass Spectrometry. Anal. Chem. 91 (17), 11355-11361.
(20) Astarita, G., Avanesian, A., Grimaldi, B., Realini, N., Justinova, Z., Panlilio, L. V., Basit, A., Goldberg, S. R., and Piomelli, D. (2015) Methamphetamine accelerates cellular senescence through stimulation of de novo ceramide biosynthesis. PLoS One 10 (2), No. e0116961.

(21) Ladd, S. L., Sommer, S. A., LaBerge, S., and Toscano, W. (1993) Effect of phosphatidylcholine on explicit memory. Clin. Neuropharmacol. 16 (6), 540-549.

(22) Whiley, L., Sen, A., Heaton, J., Proitsi, P., Garcia-Gomez, D., Leung, R., Smith, N., Thambisetty, M., Kloszewska, I., Mecocci, P., Soininen, H., Tsolaki, M., Vellas, B., Lovestone, S., and LegidoQuigley, C. (2014) Evidence of altered phosphatidylcholine metabolism in Alzheimer's disease. Neurobiol. Aging 35 (2), 271-278.

(23) Blusztajn, J. K., Slack, B. E., and Mellott, T. J. (2017) Neuroprotective Actions of Dietary Choline. Nutrients 9 (8), 815.

(24) McDonald, B. C., Flashman, L. A., Arciniegas, D. B., Ferguson, R. J., Xing, L., Harezlak, J., Sprehn, G. C., Hammond, F. M., Maerlender, A. C., Kruck, C. L., Gillock, K. L., Frey, K., Wall, R. N., Saykin, A. J., and McAllister, T. W. (2017) Methylphenidate and Memory and Attention Adaptation Training for Persistent Cognitive Symptoms after Traumatic Brain Injury: A Randomized, PlaceboControlled Trial. Neuropsychopharmacology 42 (9), 1766-1775.

(25) Ramasubbu, R., and Goodyear, B. G. (2008) Methylphenidate modulates activity within cognitive neural networks of patients with post-stroke major depression: A placebo-controlled fMRI study. Neuropsychiatr Dis Treat 4 (6), 1251-1266.

(26) Curatolo, P., Paloscia, C., D’Agati, E., Moavero, R., and Pasini, A. (2009) The neurobiology of attention deficit/hyperactivity disorder. Eur. J. Paediatr Neurol 13 (4), 299-304.

(27) Goldstein, R. Z., Woicik, P. A., Maloney, T., Tomasi, D., AliaKlein, N., Shan, J., Honorio, J., Samaras, D., Wang, R., Telang, F., Wang, G. J., and Volkow, N. D. (2010) Oral methylphenidate normalizes cingulate activity in cocaine addiction during a salient cognitive task. Proc. Natl. Acad. Sci. U. S. A. 107 (38), 16667-16672.

(28) Amatore, C., Arbault, S., Bouret, Y., Guille, M., Lemaitre, F., and Verchier, Y. (2006) Regulation of exocytosis in chromaffin cells by trans-insertion of lysophosphatidylcholine and arachidonic acid into the outer leaflet of the cell membrane. ChemBioChem 7 (12), 1998-2003.

(29) Uchiyama, Y., Maxson, M. M., Sawada, T., Nakano, A., and Ewing, A. G. (2007) Phospholipid mediated plasticity in exocytosis observed in PC12 cells. Brain Res. 1151, 46-54.

(30) Ross, B. M., and Turenne, S. D. (2002) Chronic cocaine administration reduces phospholipase $\mathrm{A}(2)$ activity in rat brain striatum. Prostaglandins, Leukotrienes Essent. Fatty Acids 66 (5-6), 479-483.

(31) Farooqui, A. A., Horrocks, L. A., and Farooqui, T. (2000) Glycerophospholipids in brain: their metabolism, incorporation into membranes, functions, and involvement in neurological disorders. Chem. Phys. Lipids 106 (1), 1-29.

(32) Wang, H., Treadway, T., Covey, D. P., Cheer, J. F., and Lupica, C. R. (2015) Cocaine-Induced Endocannabinoid Mobilization in the Ventral Tegmental Area. Cell Rep. 12 (12), 1997-2008.

(33) Carrasco, G. A., and Battaglia, G. (2007) Withdrawal from a single exposure to cocaine increases 5-HT2A receptor and G protein function. NeuroReport 18 (1), 51-55.

(34) Franklin, J. M., and Carrasco, G. A. (2015) Cocaine potentiates multiple 5-HT2A receptor signaling pathways and is associated with decreased phosphorylation of 5-HT2A receptors in vivo. J. Mol. Neurosci. 55 (3), 770-777.

(35) Simon, S. L., Dacey, J., Glynn, S., Rawson, R., and Ling, W. (2004) The effect of relapse on cognition in abstinent methamphetamine abusers. J. Subst Abuse Treat 27 (1), 59-66.

(36) Quednow, B. B., Jessen, F., Kuhn, K. U., Maier, W., Daum, I., and Wagner, M. (2006) Memory deficits in abstinent MDMA (ecstasy) users: neuropsychological evidence of frontal dysfunction. J. Psychopharmacol. 20 (3), 373-384.

(37) Calipari, E. S., Ferris, M. J., Siciliano, C. A., and Jones, S. R. (2015) Differential influence of dopamine transport rate on the 
potencies of cocaine, amphetamine, and methylphenidate. ACS Chem. Neurosci. 6 (1), 155-162.

(38) Zhu, W., Gu, C., Dunevall, J., Ren, L., Zhou, X., and Ewing, A. G. (2019) Combined Amperometry and Electrochemical Cytometry Reveal Differential Effects of Cocaine and Methylphenidate on Exocytosis and the Fraction of Chemical Release. Angew. Chem., Int. Ed. 58 (13), 4238-4242.

(39) Volz, T. J., Farnsworth, S. J., King, J. L., Riddle, E. L., Hanson, G. R., and Fleckenstein, A. E. (2007) Methylphenidate administration alters vesicular monoamine transporter- 2 function in cytoplasmic and membrane-associated vesicles. J. Pharmacol. Exp. Ther. 323 (2), 738745 .

(40) Berglund, E. C., Makos, M. A., Keighron, J. D., Phan, N. T. N., Heien, M. L., and Ewing, A. G. (2013) Oral administration of methylphenidate blocks the effect of cocaine on uptake at the Drosophila dopamine transporter. ACS Chem. Neurosci. 4 (4), 566574.

(41) Kuczenski, R., and Segal, D. S. (2002) Exposure of adolescent rats to oral methylphenidate: preferential effects on extracellular norepinephrine and absence of sensitization and cross-sensitization to methamphetamine. J. Neurosci. 22 (16), 7264-7271.

(42) Fletcher, J. S., Rabbani, S., Henderson, A., Blenkinsopp, P., Thompson, S. P., Lockyer, N. P., and Vickerman, J. C. (2008) A new dynamic in mass spectral imaging of single biological cells. Anal. Chem. 80 (23), 9058-9064. 